\title{
Pregnancy exacerbates complications of acquired hemophilia in a patient with systemic lupus erythematosus
}

\author{
Agata Sebastiann ${ }^{1}$, Maria Misterska-Skóra ${ }^{1}$, Maria Podolak-Dawidziak², Magdalena Szmyrka-Kaczmarek ${ }^{1}$, \\ Maciej Sebastian ${ }^{3}$, Piotr Wiland ${ }^{1}$
}

\author{
'Department of Rheumatology and Internal Medicine, Wroclaw Medical University, Wroclaw, Poland \\ Head of the Department: Prof. Piotr Wiland MD, PhD \\ 2Department of Hematology and Bone Marrow Transplantation, Wroclaw Medical University, Wroclaw, Poland \\ Head of the Department: Prof. Kazimierz Kuliczkowski MD, PhD \\ ${ }^{3}$ Department of Minimally Invasive Surgery and Proctology, Wroclaw Medical University, Wroclaw, Poland \\ Head of the Department: Prof. Jerzy Rudnicki MD, PhD
}

Postep Derm Alergol 2015; XXXII (3): 235-238

DOI: 10.5114/pdia.2014.40964

Acquired hemophilia $(\mathrm{AH})$ is a rare disease characterized by the production of antibodies directed against blood coagulation fVIII leading to coagulation disorders due to lowering of its activity. These are mostly the IgG class of antibodies which does not fixate the complement (IgG1 and IgG4 class) against A2 or C2 domains of fVIII which are responsible for its activity. The incidence of $\mathrm{AH}$ is $1.3-1.5$ cases per 1000000 population per year and may affect both women and men [1]. Acquired hemophilia may be a primary disease or occur in the course of malignant diseases (e.g. prostate and lung cancer, lymphoproliferative and myeloproliferative syndromes), autoimmune diseases, during drug therapy (e.g. penicillin, sulfonamides, phenytoin, chloramphenicol, methyldopa, fludarabine and interferon) and pregnancy and puerperium. There were reported cases of $\mathrm{AH}$ in the course of systemic lupus erythematosus (SLE), primary Sjögren's syndrome, rheumatoid arthritis, giant cell arteritis and polymyositis. In around $50 \%$ of cases the exact cause is unknown [2, 3]. Acquired hemophilia can occur at any age and is characterized by hemorrhagic diathesis caused by bleeding into soft tissues, skin and mucosa. Other symptoms include large areas of ecchymoses, hematomas under the skin, nosebleed, hemoptysis, vaginal, urinal, gastrointestinal and retroperitoneal bleeding. In contrast to congenital hemophilia, hemarthroses are rare [2]. Laboratory tests reveal prolonged activated partial thromboplastin time (APTT), which is not corrected by infusion of fresh frozen plasma (FFP) and prothrombin time (PT) within normal range. Acquired hemophilia is usually characterized by a severe course of the disease and a high mortality rate $[4,5]$.
A 25-year-old woman with suspected antiphospholipid syndrome (APS) in the course of SLE was admitted to the Department of Rheumatology and Internal Medicine in September 2010 to assess the disease course and treatment options. A detailed history revealed recurrent episodes of urticaria and peripheral arteritis since 2007. Since then, she has been under the dermatologist's supervision and laboratory tests revealed antinuclear antibodies (ANA), decreased levels of both complement C3 and C4 components, normocytic anemia and leukopenia. The diagnosis of SLE was established on the basis of both clinical and laboratory findings and glucocorticoid (GC) therapy was introduced. The treatment consisted of five pulses of intravenous methylprednisolone (4000 mg between 01.2008 and 09.2008) and then between 01.2008 and 07.2009 of oral prednisone (60-10 mg/day) and since 07.2009 of oral deflazacort $12 \mathrm{mg} /$ day without evidence of clinical improvement. Additionally, there was used azathioprine (100 mg/day for 1 month, 03.2008) which was withdrawn due to elevations in serum liver transaminases and oral cyclophosphamide $100 \mathrm{mg} /$ day (6 months, until 02.2010, in a total dose of $15 \mathrm{~g}$ ) which was withdrawn due to increasing leucopenia and hair loss.

The exacerbation of joint symptoms started in August 2010 and was associated with dry cough and an episode of hemoptysis and increasing dyspnea. There also appeared multiple areas of ecchymoses on the upper and lower extremities. Pulmonary embolism (PE) was diagnosed on the basis of elevated levels of D-dimer and peripheral filling defect in an artery in segment 9 of the left lung detected by the spiral computed angiotomography of the chest. Laboratory testing revealed prolonged

Address for correspondence: Agata Sebastian, Department of Rheumatology and Internal Medicine, Wroclaw Medical University, 213 Borowska St, 50-556 Wroclaw, Poland, phone: +48 71734 33 50, +48 515128 306, fax: +48 7173433 09, e-mail: agatasebastian@vp.pl Received: 5.12.2013, accepted: 2.01.2014. 
APTT (100 s, normal value $25-37$ s), leukopenia $(2.51 \times$ $10^{3} / \mu \mathrm{l}$, normal value $4-10 \times 10^{3} / \mu \mathrm{l}$ ), anemia (hemoglobin level $7.2 \mathrm{~g} / \mathrm{dl}$, normal value $12-16 \mathrm{~g} / \mathrm{dl}$ ), elevated levels of $\mathrm{C}$-reactive protein and the platelet (PLT) count of $210 \times 10^{3} / \mu \mathrm{l}\left(\right.$ normal value $\left.140-440 \times 10^{3} / \mu \mathrm{l}\right)$. The treatment introduced by dermatologists consisted of low molecular weight heparin (LMWH) (nadroparin calcium) $0.6 \mathrm{ml} 2 x /$ day (in spite of unexplained prolonged APTT) and 2 units of red blood cells and 2 units of FFP were transfused.

The patient was admitted to the Department of Rheumatology in September 2010 with large and multiple areas of ecchymoses in the upper and lower extremities

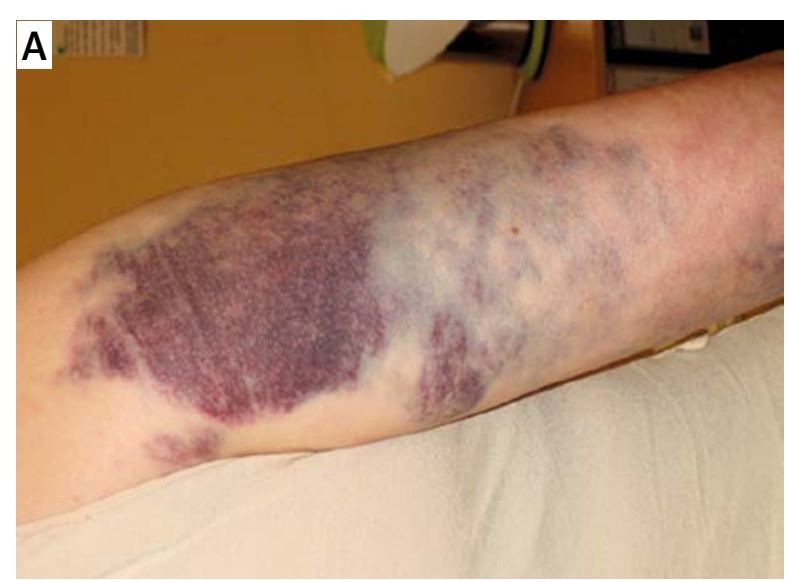

B

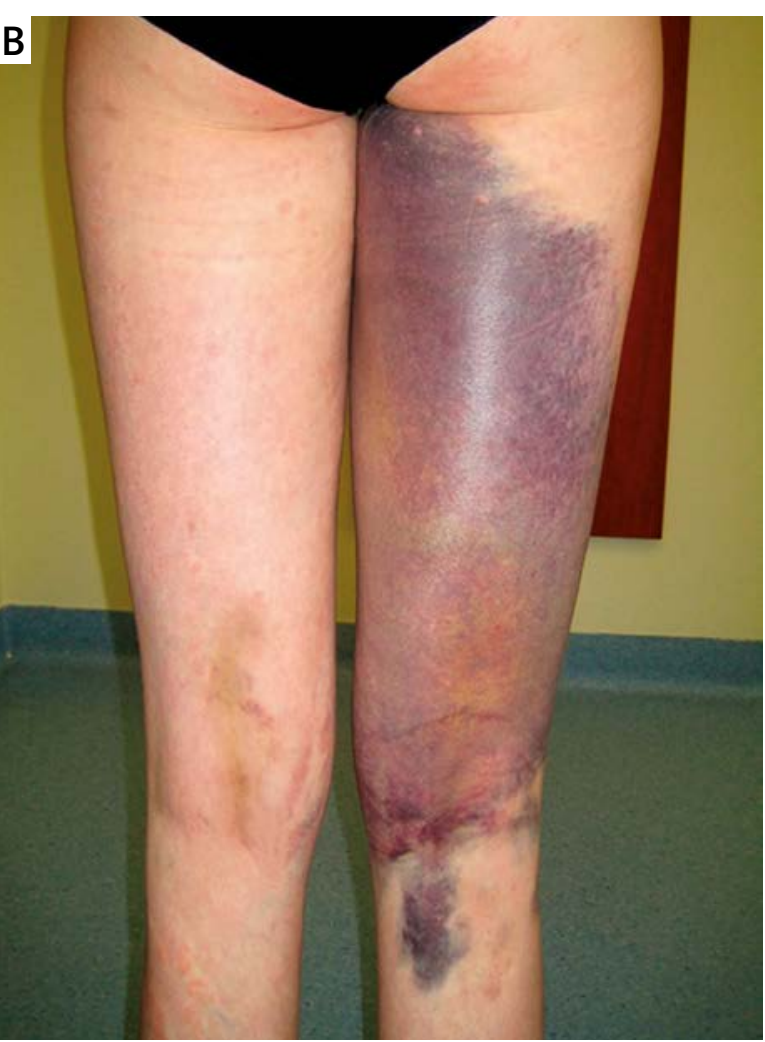

Figure 1. Ecchymoses: $\mathbf{A}$ - on the upper extremity, $\mathbf{B}$ - on the lower extremity
(Figures $1 \mathrm{~A}, \mathrm{~B}$ ). During hospitalization there appeared bilateral episcleritis which was treated topically and recurring skin rash in the form of urticaria (Figure 2). Laboratory testing revealed significantly prolonged APTT (121 s, normal value $25-37 \mathrm{~s})$, leukopenia $\left(3.33 \times 10^{3} / \mu \mathrm{l}\right.$, normal value $\left.4-10 \times 10^{3} / \mu \mathrm{l}\right)$, lymphopenia $\left(0.93 \times 10^{3} / \mu \mathrm{l}\right.$, normal value $1.5-3.5 \times 10^{3} / \mu \mathrm{l}$ ), anemia (hemoglobin level 10.9 $\mathrm{g} / \mathrm{dl}$, normal value $12-16 \mathrm{~g} / \mathrm{dl}$ ), PLT count of $205 \times 10^{3} /$ $\mu \mathrm{l}$ (normal value $\left.140-440 \times 10^{3} / \mu \mathrm{l}\right)$, decreased levels of both complement C3 $(0.57$, normal value $0.9-1.8)$ and C4 ( $<0.08$, normal value 0.1-0.4) components, the presence of antinuclear antibodies (ANA) $(1: 320)$ which exhibited a granular pattern of fluorescence and SS-A and Ro52 antibodies. The level of ds-DNA was $18.35 \mathrm{IU} / \mathrm{ml}$ (normal value $<100)$. The APS and PE were excluded on the basis of laboratory and immunological testing (lupus anticoagulant (LA), antibodies against $\beta_{2}$-glycoprotein (anti- $\beta_{2} \mathrm{GPI}$ ), anticardiolipin antibodies (ACA) ) and a control spiral computed angiotomography of the chest. Treatment with LMWH was stopped. A slight reduction in APTT to $115 \mathrm{~s}$ was achieved. Physical examination revealed a smaller number and size of areas of ecchymoses. The diagnosis of $\mathrm{AH}$ was established on the basis of low coagulation fVIII level (<5\%, normal value 50-150\%). The level of coagulation $\mathrm{fVIII}$ inhibitor measured with Bethesda method was $614.4 \mathrm{lU} / \mathrm{ml}$ (the Bethesda units are used to evaluate the concentration of fVIII inhibitor what is based on establishing such dilution when the activity of fVIII is $50 \%$. The higher concentration of the inhibitor, the higher the number of Bethesda units). The treatment consisted of oral methylprednisolone $20 \mathrm{mg} /$ day, chloroquine $250 \mathrm{mg} /$ day and cyclosporine $200 \mathrm{mg} /$ day what led to clinical improvement. A prolonged APTT was still observed in laboratory tests.

Five months later the patient was 7 weeks' pregnant. In the $10^{\text {th }}$ week of gestational age, the retroperitoneal bleeding (in the lumbar region and on both sides of the uterus) was observed by transvaginal and abdominal ultrasound imaging. The level of APTT was $94 \mathrm{~s}$ (normal

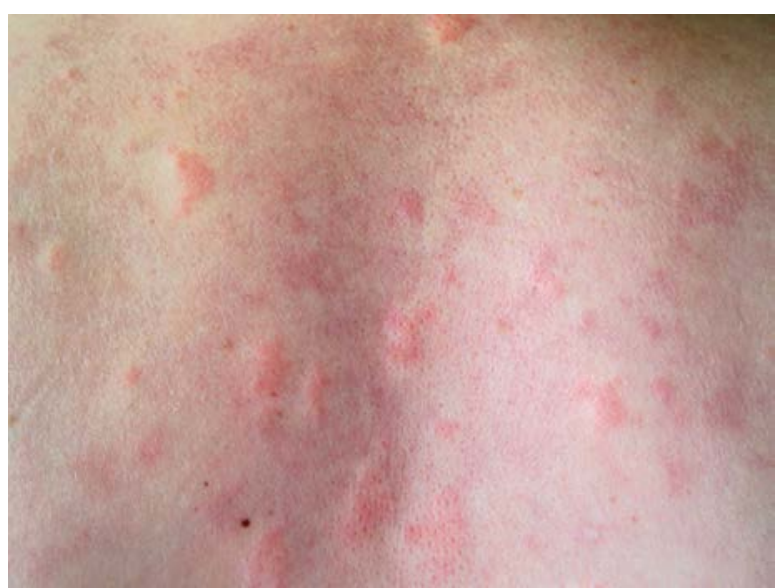

Figure 2. Urticaria on the back 
value $25-37$ s), fVIII 1.33\% (normal value 50-150\%), hemoglobin $-5.2 \mathrm{~g} / \mathrm{dl}$ (normal value $12-16 \mathrm{~g} / \mathrm{dl})$, and PLT count of $170 \times 10^{3} / \mu \mathrm{l}$ (normal value $140-440 \times 10^{3} / \mu \mathrm{l}$ ). Glucocorticoid and cyclosporine therapy was maintained. After getting written consent from the patient, the pregnancy was terminated under the protection of recombinant factor VII ( $\mathrm{rVIII}$ ). The treatment of the disease consisted of methylprednisolone i.v., then the prednisone $1 \mathrm{mg} / \mathrm{kg}$ and the dose of cyclosporine was increased to $250 \mathrm{mg} /$ day. The rfVII was continued until the coagulation parameters were within the normal range. The treatment resulted in clinically meaningful improvement and normalization of laboratory tests were noted. The remission was established after withdrawal of rfVII and the symptoms of hemorrhagic diathesis were not observed. The level of APTT was within the normal range in control laboratory testing and the antibodies against fVIII were not detected until now. Chloroquine $250 \mathrm{mg}$ / day, cyclosporine $200 \mathrm{mg} /$ day and methylprednisolone in a maintenance dose were maintained in the therapy.

The most common hematologic complication of SLE associated with the blood coagulation system is APS characterized by the presence of anti- $\beta_{2} \mathrm{GPI}$ antibodies, ACA and LA [2]. Thus, our patient was initially diagnosed with PE in the course of APS and treated with LMWH. Initially, the petechiae were believed to be associated with heparin treatment. Laboratory tests revealed significantly prolonged APTT and antibodies typical of APS were assessed. In patients treated with $L M W H$, the results of LA testing may be inadequate. It is not recommended to assess this parameter during thrombotic episodes due to the presence of acute inflammatory reactions (including fVIII) what may influence the APTT results. Lupus anticoagulant testing was performed twice during and after withdrawal of LMWH treatment and neither the presence of anti- $\beta_{2}$ GPI nor LA were confirmed. The LMWH treatment caused the hemorrhagic diathesis which did not disappear after its withdrawal. The clinical manifestations consisting of dry cough with an episode of hemoptysis and increasing dyspnea should be associated with the upper respiratory tract infection concomitant with hemorrhagic diathesis because the PE was excluded on the basis of the second spiral computed angiotomography of the chest.

There were reported cases of $\mathrm{AH}$ in the course of SLE in pregnancy and puerperium in both active and inactive SLE [6]. The patient got pregnant despite the fact that effective contraception was recommended and retroperitoneal bleeding occurred. Pregnancy in patients with $\mathrm{AH}$ is particularly dangerous to both the mother and the fetus because the hemorrhage can occur not only in the mother but antibodies against coagulant factors can pass through the placental barrier and lead also to massive bleeding in the fetus [6]. In our case, the pregnancy occurred after the diagnosis of $\mathrm{AH}$ what worsened the course of both SLE and AH.
The treatment of $\mathrm{AH}$ consists of two parallel strategies: the temporary control of bleeding and removal of coagulation inhibitors [4]. Desmopressin (DDAVP) or the fVIII concentrate may be used to control bleeding in patients with inhibitor concentration $<5$ Bethesda units and moderate bleeding. The infusions of rfVIla (NovoSeven, at a dose of $90 \mu \mathrm{g} / \mathrm{kg}$ i.v. every $2 \mathrm{~h}$ until bleeding cessation, then more rarely) or the activated prothrombin complex also known as the anti-inhibitor coagulant complex (AICC) (at a dose of $75 \mathrm{U} / \mathrm{kg}$ i.v. every 8-12 h) may be used in patients with a higher concentration of the inhibitor. Immunosuppressive or immunomodulatory therapies are used to inhibit further synthesis of coagulation inhibitors. Treatment consists of a high dose GC as the first-line therapy e.g. prednisone at a dose of $1 \mathrm{mg} / \mathrm{kg}$ for several weeks with a further dose reduction. It was found that combined therapy with prednisone plus cyclophosphamide at an oral dose of $2 \mathrm{mg} / \mathrm{kg} /$ day may be effective in patients with no response to steroid monotherapy [2, 4, 7-9]. Other immunosuppressive drugs may be used e.g. azathioprine, vincristine or cyclosporine A [5]. Rituximab monotherapy may be also effective at a dose of $375 \mathrm{mg} / \mathrm{kg}$ i.v. once a week for 4 weeks [10, 11]. Other possible treatment options include plasmapheresis and immunoadsorption [4].

Our case is interesting for several reasons. Firstly, at the beginning of the disease the diagnosis of APS complicated with PE was very probable due to its symptoms (dyspnea, hemoptysis, chest pain) with prolonged APTT and characteristic features in spiral computed angiotomography of the chest. Antiphospholipid syndrome is a more common complication of SLE than AH because antiphospholipid antibodies are found in about $30 \%$ of patients with SLE. The prolonged APTT can be associated not only with LA, but also with more infrequent presence of coagulation inhibitors. In some patients, coagulation inhibitors may be concomitant with antiphospholipid antibodies [2]. Secondly, most of the published reported cases of acquired hemophilia associated with lupus erythematosus are females in the child-bearing age (9 of 12, range 19-45 years) [2], thus effective contraception and patient compliance are very important issues.

In conclusion, APTT should be monitored in patients with SLE associated with symptomatic hemorrhagic diathesis. If the APTT is prolonged in the absence of LA, the $\mathrm{AH}$ should be suspected and further tests should be done to confirm the diagnosis. It is very important to notice worrying symptoms as fast as possible and treat patients with $\mathrm{AH}$ in collaboration with hematology centers to prevent incidents of life-threatening bleeding.

\section{Acknowledgments}

The work was conducted and should be attributed to the Department of Rheumatology and Internal Medicine, 
Department of Hematology and Bone Marrow Transplantation and Department of Minimally Invasive Surgery and Proctology, Wroclaw Medical University.

\section{Conflict of interest}

The authors declare no conflict of interest.

\section{References}

1. Piś Mital A. Nabyte inhibitory krzepnięcia. Acta Haematol Pol 2009; 40: 26-31.

2. Rezaieyazdi Z, Sharifi-Doloui D, Hashemzadeh K, et al. Acquired haemophilia $A$ in a woman with autoimmune hepatitis and systemic lupus erythematosus; review of literature. Blood Coagul Fibrinolysis 2012; 23: 71-4.

3. Klein KG, Parkin JD, Madaras F. Studies on an acquired inhibition of factor VIII induced by penicillin allergy. Clin Exp Immunol 1976; 26: 155-61.

4. Akahoshi M, Lizawa K, Nagano S, et al. Acquired hemophilia in a patient with systemic lupus erythematosus: a case report and literature review. Mod Rheumatol 2008; 18: 511-5.

5. Schulman S, Langevitz P, Livneh A, et al. Cyclosporine therapy for acquired factor VIII inhibitor in a patient with systemic lupus erythematosus. Thromb Haemost 1996; 76: 344-6.

6. Tengborn L, Baudo F, Huth-Kühne A, et al. Pregnancy-associated acquired haemophilia $A$ : results from the European Acquired Haemophilia (EACH2) registry. BJOG 2012; 119: 1529-37.

7. Porru G, Mura V, Piga M, et al. Hemarthrosis as acute presentation of acquired hemophilia in a patient with systemic lupus erythematosus: successful treatment and long-lasting remission. Clin Rheumatol 2008; 27: 1581-4.

8. Knoebl P, Marco P, Baudo F, et al. Demographic and clinical data in acquired hemophilia A: results from the European Acquired Haemophilia Registry (EACH2). J Thromb Haemost 2012; 10: 622-31.

9. Delgado J, Jimenez-Yuste V, Hernandez-Navarro F, et al. Acquired haemophilia: review and meta-analysis focused on therapy and prognostic factors. Br J Haematol 2003; 121: 21-35.

10. Kain S, Copeland TS, Leahy MF. Treatment of refractory autoimmune (acquired) haemophilia with anti-CD20 (rituximab). Br J Haematol 2002; 119: 578.

11. Wiestner A, Cho HJ, Asch AS, et al. Rituximab in the treatment of acquired factor VIII inhibitors. Blood 2002; 100: 3426-8. 